\title{
Serial-position curves for reaction time and accuracy in visual search: Tests of a model of overlapping processing
}

\author{
JUDITH RICH HARRIS \\ Middletown, New Jersey \\ MARILYN L. SHAW \\ AT\&T Bell Laboratories, Murray Hill, New Jersey, and Rutgers University, New Brunswick, New Jersey
}

and

MARY JO ALTOM

AT\&T Bell Laboratories, Murray Hill, New Jersey

\begin{abstract}
In 1979, Harris, Shaw, and Bates presented a new model for visual search in briefly presented multicharacter displays. The model was based on the assumption that processing of characters is neither serial nor parallel, but overlaps in time. This model of overlapping processing generates predicted serial-position curves for both reaction time and accuracy. For short arrays, the curves are monotonic; for longer arrays, the curves bend over at the end-predicted performance for the last item is better than for the next-to-last. An experiment designed as a test of the overlapping model produced serial-position curves that matched the model's predictions quite well: the model accounts for $98 \%$ of the variance in the reaction time data and $95 \%$ of the variance in the accuracy data. The assumption that processing of individual array items overlaps in time can account for results (such as interference between adjacent array items) that suggest simultaneous processing. It can also account for results suggesting that items in an array are processed in a particular order. The same assumption can, moreover, explain previous results that match neither serial nor parallel predictions, but fall somewhere between the two.
\end{abstract}

In 1979, Harris, Shaw, and Bates introduced a new model for visual search, a model of overlapping processing. The overlapping model was developed because earlier models, including Shaw's (1978) capacity allocation model, had proven inadequate to account for several phenomena found in visual search experiments. In particular, neither serial nor parallel models could account for the reaction time (RT) data from the first experiment reported in Harris et al., which will be referred to here as the gap experiment.

The stimuli in the gap experiment were briefly flashed arrays of 10 letters, arranged in a horizontal string. Some of the arrays contained gaps-three consecutive missing letters. The subjects' task was to judge which of two possible target letters was present in each array. The target

The experiment and some of the analyses reported in this paper were carried out at Rutgers University, supported by Grant 5 R01 HD-1387802 from the U.S. Department of Health, Education and Welfare. Additional analyses and manuscript preparation were supported by Grant BNS 83-08610 from the National Science Foundation and by the Human Information Processing Research Department at AT\&T Bell Laboratories. Marilyn L. Shaw, the principal investigator on the two grants, died in November 1983. Address correspondence to Judith Rich Harris, 54 Crawford Road, Middletown, NJ 07748. We thank Saul Sternberg for his many suggestions and comments on earlier drafts of this paper. appeared in either the third, fifth, or seventh position from the left; when gaps were present they were located either to the left or to the right of targets in positions 5 and 7. The subjects were instructed to fixate a point at the left end of the display and to process ("read") the letters from left to right.

Harris et al. found that displays with gaps produced significantly faster and more accurate responses than displays without gaps, and that a gap to the right of the target (after the target in a left-to-right processing order) produced significantly faster and more accurate responses than did a gap to its left.

Although both serial and parallel models would generate a prediction of improved performance in arrays with gaps, neither type of model could account for the specific characteristics of gap effects. With a self-terminating serial model (Sternberg, 1967), a gap before the target should help, because the missing letters would not have to be processed. However, a gap after the target should have no effect. With an exhaustive serial model, a gap anywhere in the array should lead to the same amount of improvement. A parallel model might account for the improved performance produced by a gap by the absence of lateral interference that the missing letters would have produced. However, parallel models cannot account for 
the fact-shown by Estes and Wolford (1971) for accuracy - that the asymmetry of the gap effect depends highly on instructed processing order (the order in which subjects are told to process the stimuli, or to report them). Other factors, such as retinal locus, do not have as great an effect as instructed processing order.

Instructed processing order also matters more than retinal locus in determining the serial-position effect (Estes \& Wolford, 1971; Shaw, 1977). The serial-position effect is the tendency for both speed and accuracy of response to a stimulus to depend on its ordinal position in an array of stimuli. It is an important and familiar phenomenon in information-processing experiments. Performance is almost invariably best for the first item in the instructed processing order, regardless of its retinal position. For small arrays-no more than five or six characters-performance tends to worsen steadily as ordinal position increases. Data for larger arrays have been obtained in whole-report accuracy experiments (experiments in which the subjects' task is to report as many characters as they can recall from the array). These data indicate that the serial-position effect for larger arrays is not monotonic: it is somewhat U-shaped, with performance for the last character in the processing order being better than that for the next-to-last. The dependence of the serial-position effect on instructed processing order is a serious problem for parallel models.

Harris et al. proposed that processing of array items is accomplished in neither a purely serial nor a purely parallel manner. Their model is based on the assumption that processing of successive characters overlaps in time: items are scanned consecutively and enter the processor in a given order, but the rate of the scan is much faster than the rate of processing. Thus, processing of the second item (and then the third, and so on) begins before the first is completed. The result is that several items are being processed at once, but they have entered the processor one at a time, in serial order. Gap effects and serialposition effects are accounted for by the assumption that the processor deals with each of several items somewhat less efficiently than it would deal with one alone. The first and last items in the processing order suffer least from competition for processing capacity; items next to a gap are benefited by a brief period during which no new items enter the processor, since the scanning mechanism moves at a constant rate across the display. Items surrounded by several other items suffer the most from competition for processing capacity.

The tendency for a given character to interfere in some way with the detection of adjacent characters has been referred to as "lateral interference" or "lateral masking." In our view, these terms are not an explanation at all, but merely a description of an observed phenomenon. We account for this phenomenon by assuming that it is due to the competition for processing capacity that occurs during the time when adjacent characters are being processed simultaneously.
Although the overlapping model was described in detail in the earlier paper (Harris et al., 1979), a summary of its mathematical specifications might be useful here. The model makes quantitative predictions on the basis of the following assumptions:

(1) The scanner travels across the display at a fixed rate of $10 \mathrm{msec}$ per display position, whether a given position contains a character or an empty space. (The 10-msecper-character rate was chosen on a priori grounds, based on previous evidence; see Harris et al.) Thus, with a display that contains no gaps, a new item enters the processor every $10 \mathrm{msec}$.

(2) Processing efficiency is at its maximum, 1.00, for an item that is being processed alone. Efficiency goes down each time a new item enters the processor; it goes up each time an item completes its processing and exits from the processor. In each interval of time, $j$, between two such events, processing efficiency remains constant. The term $\xi_{i, j}$ denotes the efficiency of processing item $i$ during time interval $\mathrm{j}$.

(3) Processing efficiency, $\xi_{i, j}$, also depends on the values of two parameters, $\lambda_{\mathrm{m}}$ and $\lambda_{\mathrm{n}}$; both parameters are positive numbers between 0 and 1 . The first parameter, $\lambda_{\mathrm{m}}$, determines the effect on $\xi_{\mathrm{i}, \mathrm{j}}$ produced by the items that entered the processor before item i; the second, $\lambda_{\mathrm{n}}$, determines the effect of the items that entered after item i. The relationship between these two parameters and processing efficiency is:

$$
\xi_{i, j}=\left[\lambda_{m}{ }^{\log _{e}\left(m_{i, j}+1\right)}\right]\left[\lambda_{n} \log _{e}\left(n_{i, j}+1\right)\right],
$$

where $m_{i, j}$ is the number of items that entered the processor before item $i$ and are still being processed during interval $j$, and $n_{i, j}$ is the number that entered after item $i$ and are also being processed during interval $j$. The values assigned to $\lambda_{m}$ and $\lambda_{n}$ by a least squares fit to the gap data suggest that items entering after a given item interfere with its processing more than do items that entered before that item. This is in accord with the notion that processing of a given character is rapid at first and slower later on (Sperling, 1970): new items are given more processing capacity than older ones. ${ }^{1}$

(4) The amount of processing that item i receives during interval $j$ equals the length of interval $j$ times the processing efficiency during that interval. Processing of $i$ is completed when the sum of the amounts of processing accomplished during each of these intervals reaches $\tau_{\mathrm{i}}$, which we previously labeled "required processing time" but which might better be called required amount of processing. The required amount of processing for a given item depends on that item's distance from the fixation point (which increases with its serial position) and on how much decay its trace has undergone by the time it enters processing (an item that is scanned early has had less time to decay). Since retinal position and decay of trace both depend on serial position, they can be represented by a single parameter, $k$. The relationship be- 
tween $\mathrm{k}$ and required amount of processing for item $\mathrm{i}$, $\tau_{\mathfrak{i}}$, is:

$$
\tau_{\mathrm{i}}=\tau_{1} \mathrm{k}^{\mathrm{i}-1}
$$

where $i$ is the serial position and $\tau_{1}$ equals the processing time required for an item that is at the fixation point, scanned at once, and processed alone. It was assumed on a priori grounds (for reasons given in Harris et al.) that this amount of time, called "minimum encoding time," equals $40 \mathrm{msec}$.

(5) The interval of time that passes between when item i is scanned and when its processing is completed is the predicted encoding time for that item, $\mathrm{T}_{\mathrm{i}}$. Since we assume that processing ceases once the target is found, the RT prediction for a given display is determined by calculating the predicted encoding time for the display position that contains the target, and adding to that value the product $(\mathrm{i}-1)(10 \mathrm{msec})$, to allow for the amount of time that passes before that position is scanned. This sum is referred to as the predicted $R T$ increment; it represents the time required for the first stage of information processing, which has been called the encoding stage. ${ }^{2}$

With the values of $\lambda_{m}, \lambda_{n}$, and $k$ set by the fit to the gap data, the overlapping model generates predicted serial position curves for RT that are monotonic for array sizes up to five (see Figure 1). For arrays of more than six items, the curve bends over at the end-slowest RTs are predicted not for the last item, but for the next-to-last. This prediction has remained untested until now because previous visual search experiments in which RT was measured have not used displays that were large enough, or-in the case of the gap experiment-have not tested targets beyond the seventh display position.

Harris et al. also presented a version of the overlapping model that predicts accuracy rather than RT. The accuracy version was based on what is known as a deadline model of speed-accuracy tradeoff. It was assumed that processing of each array item is allotted only a limited amount of time, designated by the parameter $\mathrm{A}$. When that time is up-when the deadline is reached-processing of the item ceases. It was further assumed that accuracy of responding to item $i$ equals the proportion of the predicted encoding time actually used: that is, the amount of processing time that item $i$ received, A, divided by the amount of time that would have been needed for complete processing of that item, $T_{i}$. This simple formula ${ }^{3}$ provided a moderately good fit to the accuracy data from a second gap experiment, in which shorter display durations were used and accuracy varied over a wider range (RT was not measured). The model accounted for $84 \%$ of the variance in the accuracy data.

The ability of a modifed version of the overlapping model to deal with accuracy results was encouraging, but there are conceptual problems with the accuracy version that should not be glossed over. First, the two versions of the model, as they have been described, are mutually exclusive: the model cannot logically predict both $\mathrm{RT}$ and

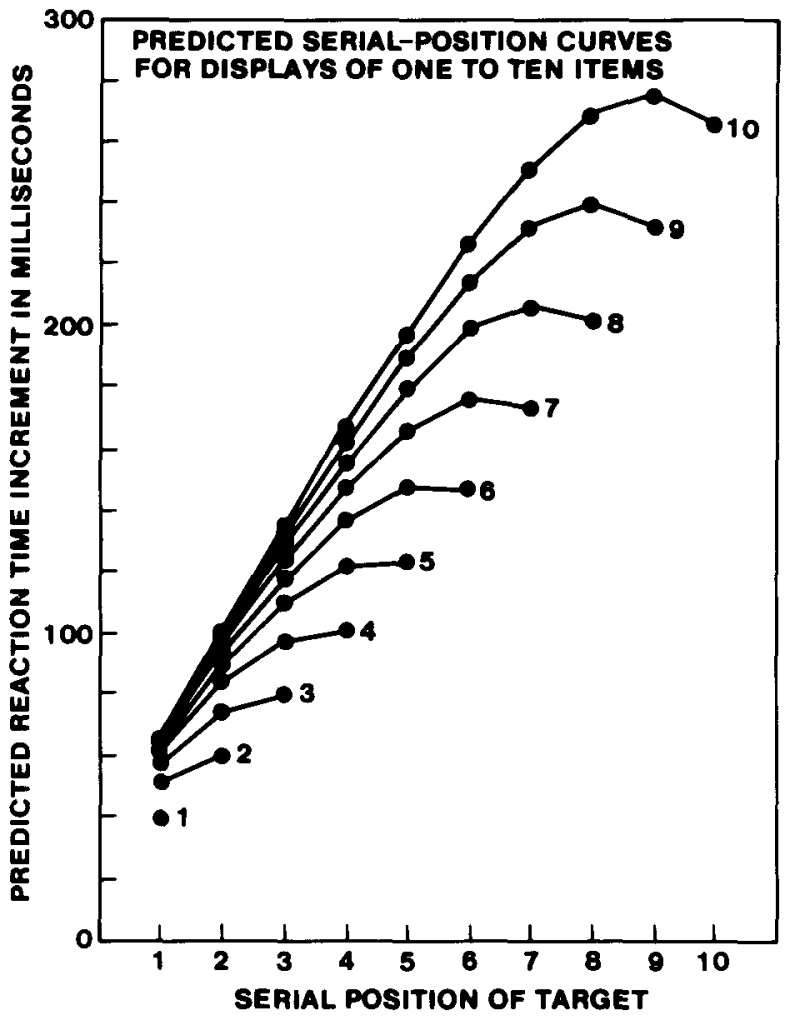

Figure 1. Predicted serial-position curves for visual search, for arrays of from one to $\mathbf{1 0}$ characters. These curves were generated by the overlapping model. Each point in this graph represents an RT prediction for a target in a given ordinal position in the array. A detailed mathematical description of the overlapping model can be found in Harris, Shaw, and Bates (1979). The parameters used to generate these predictions were obtained by a least squares fit to the gap data reported in Harris et al.; their values are $k=1.14$, $\lambda_{\mathrm{m}}=.84$, and $\lambda_{\mathrm{n}}=.63$. No RT constant (see Footnote 2 ) has been added to these predicted RTs.

accuracy for the same experiment. This is because the accuracy version of the model predicts that the processing time for every array item will be a constant, A. Thus, the accuracy version of the model would generate an implausible prediction for RT: a linear RT serial position curve with a slope close to zero. ${ }^{4}$ For the RT version of the model, on the other hand, it is assumed that each array item will be given as much processing time as it requires, $T_{i}$. Processing of each item is assumed to be complete; complete processing might be taken to mean perfect processing. Given this interpretation, the RT version of the model would generate a clearly erroneous accuracy prediction: $100 \%$ accuracy for every item.

There is another, more subtle problem with the accuracy version of the model. The model bases predicted processing times for a given item partly on the degree to which processing of that item overlaps with processing of other items. But the deadline assumption of the accuracy model implies that there is much less processing of each item than predicted by the RT model, and therefore much less overlapping. Since the model's predictions require a 
considerable amount of overlapping, the deadline assumption appears to be untenable.

Perhaps the most straightforward way out of these difficulties is to throw out the deadline assumption and reinterpret the meaning of the proportion $A / T_{i}$. Let us assume that the full amount of necessary processing time is allotted, in accordance with the RT version of the model. We can then go on to posit the simple hypothesis that accuracy varies as the reciprocal of predicted processing time, times a constant. (The constant can vary between subjects, but not within a given subject.) This hypothesis can be interpreted as follows: All processing contains some potential for error; therefore, the longer processing goes on, the more error is likely to occur. The parameter $\mathrm{A}$, then, quantifies the relationship between the length of the processing period and the increasing tendency for processing to be imperfect.

The experiment reported here was designed as a test of the overlapping model's RT and accuracy predictions. Briefly presented arrays of 10 letters were used as the stimuli, with the target appearing at random in any of the 10 positions. The subjects' task was to judge, on each trial, which of two possible target letters was present in the array.

\section{METHOD}

\section{Subjects}

The subjects were five Rutgers students, three undergraduates and two graduate students; they were paid for their participation. Two subjects were female, three were male. Each had passed a pretest designed to eliminate subjects whose corrected visual acuity was less than $20 / 20$ or whose ability to maintain fixation was inadequate.

\section{Stimuli and Apparatus}

The stimuli were horizontal strings of 10 capital letters. Each display contained one target letter, either a $D$ or an $R$. The remaining letters were Ns and Xs, randomly arranged with the constraint that no more than two consecutive letters could be the same.

The target letter appeared equally often in each of the 10 positions; it was equally likely to be a D or an R. Each block of 120 trials contained six displays for each combination of target position and target letter. These 120 displays occurred in random order, with a different random order used for each block.

Stimuli were presented on Panasonic TN-95 video monitors. Displays were controlled by a SOL microcomputer interfaced with a Digital Equipment PDP-11/40. The letters were shown in black on a gray screen; screen brightness varied among the video monitors within the range of 23 to $29 \mathrm{fL}$ ( 79 to $99 \mathrm{~cd} / \mathrm{m}^{2}$ ), as measured by a Tektronix J-16 photometer. (A given subject used the same monitor throughout the experiment.) The subjects viewed the displays through a viewing hood, which blocked off visual distractions and maintained viewing distance at $137 \mathrm{~cm}$. They made their responses by pushing one of two buttons: the button on their right indicated a response of $R$; the one on their left, a response of $D$.

The letters always appeared in the subjects' right visual field. A fixation point at the center of the screen was visible throughout the experiment. There was a horizontal space of $2.7 \mathrm{~mm}$ between the fixation point and the leftmost letter and between the proximal edges of adjacent display letters. Each letter was $2.3 \mathrm{~mm}$ wide and $4.0 \mathrm{~mm}$ high. The entire display, from fixation point through the rightmost letter, subtended $1^{\circ} 59^{\prime}$ of visual angle. Display duration was $175 \mathrm{msec}$ throughout the test sessions.

\section{Procedure}

Each subject participated in approximately five training sessions. Display duration was gradually reduced to $175 \mathrm{msec}$ during the first of these sessions. The subjects were instructed to fixate steadily on the fixation point during each trial, to "' 'read' or process the letters from left to right," and to respond as quickly and as accurately as possible. They were reminded during each training and test session of the importance of steady fixation. Feedback was given on each trial during both training and test sessions; it consisted of redisplaying the array so that the subject could see which target letter it contained. The criterion for completion of training was a minimum of $55 \%$ correct responses for each target position and a minimum of $95 \%$ for the first three target positions, at a display duration of $175 \mathrm{msec}$. Accuracy above $95 \%$ for the first three target positions and increasing RTs across the first four target positions were taken as indications of adequate eye fixation.

Each test session consisted of five blocks of 120 trials, with rest periods between blocks. Sessions lasted approximately $1.5 \mathrm{~h}$. Each subject participated in a total of at least 1,200 test trials for each target position, or at least 12,000 test trials in all. ${ }^{5}$

The trials were paced automatically by computer. Each trial consisted of the 175-msec display period, followed by a $1,500-\mathrm{msec}$ period allotted to the subject's response. This was followed by a feedback period in which the display was presented again for $1,500 \mathrm{msec}$, and a period of $1,500 \mathrm{msec}$ during which the screen was blank. The blank screen served as a warning signal that the next trial was about to begin.

\section{RESULTS}

\section{Reaction Time}

The solid lines in Figure 2 show the RT results. Each point on these serial-position curves represents mean RT to a target in the corresponding display position. Only correct responses were used to calculate these mean RTs. The first five graphs, $2 \mathrm{a}$ through $2 \mathrm{e}$, show data from the individual subjects; $2 \mathrm{f}$ shows the group data, averaged across the five subjects.

The dotted lines in the first five graphs of Figure 2 are RT serial-position curves generated by the overlapping model. The model parameters and RT constants (see Footnote 2) used to produce these predicted curves were derived from a least squares fit to each subject's data, using a grid-search procedure. The values of the parameters are given in Table 1. This table also shows the percentages of the variance accounted for by the RT model; the percentages ranged from $90 \%$ to just under $100 \%$ for the individual subjects.

We averaged the predicted curves derived from the fits to the individual subjects' data to produce a mean predicted curve, just as we averaged the data themselves to produce a mean obtained curve. The results are shown by the dotted line in Figure $2 \mathrm{f}$. The mean predicted curve accounts for $97.9 \%$ of the variance in the group RT data; standard error of prediction ${ }^{6}$ is $11 \mathrm{msec}$.

Because the model is nonlinear, fitting a generated curve directly to the group data (which are arithmetic means) is a questionable procedure. However, since this is a method often used in the past, we carried it out on the present data. The results were very similar to those shown in Figure $2 \mathrm{f}$; the curve generated by the model accounts for $98.5 \%$ of the variance in the group data. 


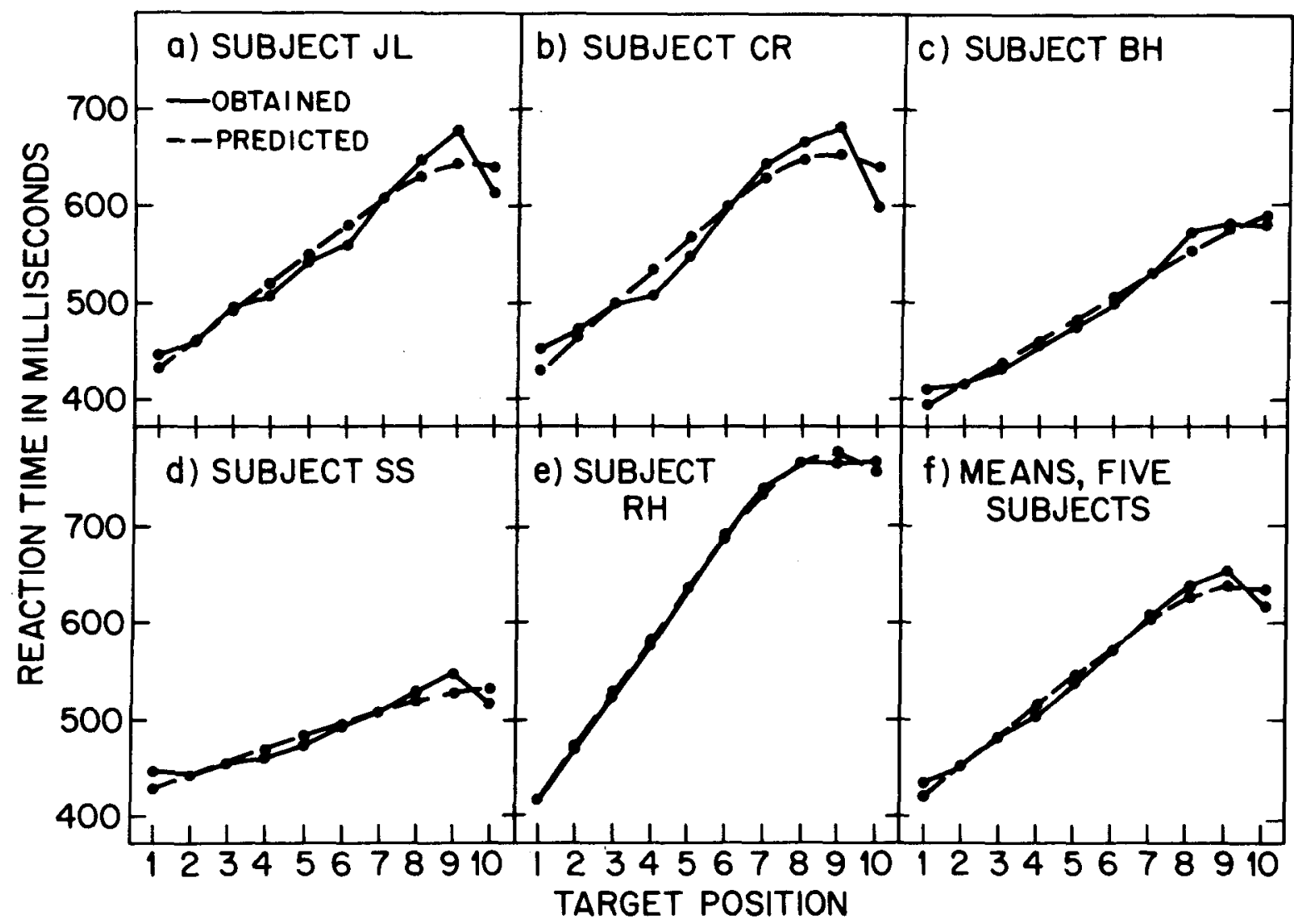

Figure 2. The solid lines in these graphs show obtained RT serial-position curves. Each point on these curves represents mean RT to targets in each of the 10 display positions. The first five graphs (2a-2e) give the data for the individual subjects; the last graph (2f) shows group performance, averaged across the five subjects. The broken lines in the first five graphs are curves generated by the overlapping model; the values of the parameters used to generate these curves, as well as the percentage of variance they account for, are given in Table 1. The broken line in the last graph, $2 \mathrm{f}$, is the mean of the five predicted curves shown in $2 \mathrm{a}-2 \mathrm{e}$. This curve accounts for $98 \%$ of the variance in the group data.

Figure 3 shows mean RT data for correct responses only (solid line) compared with means for incorrect responses only (dotted line) and with all responses, both correct and incorrect (broken line). The incorrect RTs, like the correct ones, tended to increase with target position. RTs for incorrect responses tended to be slower than the correct responses to the same target position, and to have a higher variance. Note that the means for incorrect responses are based on far fewer trials than are the means

Table 1

Results of Model-Fitting to Data from Individual Subjects

\begin{tabular}{|c|c|c|c|c|c|c|c|}
\hline \multirow[b]{2}{*}{ Subject } & \multicolumn{5}{|c|}{$\begin{array}{l}\text { Parameters Determined } \\
\text { by Least Squares Fits }\end{array}$} & \multicolumn{2}{|c|}{$\begin{array}{l}\text { Percentage of Variance } \\
\text { Accounted for by Model }\end{array}$} \\
\hline & RT Const. & $\mathbf{k}$ & $\lambda_{m}$ & $\lambda_{n}$ & A & $\mathrm{RT}$ & Accuracy \\
\hline J.L. & 374 & 1.16 & .91 & .68 & 161 & 94.7 & 91.2 \\
\hline C.R. & 360 & 1.17 & .92 & .61 & 165 & 92.5 & 88.8 \\
\hline B.H. & 349 & 1.14 & .88 & .84 & 92 & 97.8 & 94.4 \\
\hline S.S. & 380 & 1.05 & .99 & .79 & 55 & 90.0 & 69.2 \\
\hline R.H. & 339 & 1.20 & .77 & .57 & 222 & 99.7 & 96.3 \\
\hline
\end{tabular}

for correct responses; this is especially true for the early target positions (see accuracy results, below).

The combined RT data for correct and incorrect responses, shown by the broken line in Figure 3, do not differ in any important way from the RT data for correct responses alone, ${ }^{7}$ except for a slight increase in slope. This statement holds true for the data from the individual subject, as well as for the overall means. (The data for the five subjects and the 10 target positions, including RT means for correct responses, incorrect responses, and all responses, as well as percent correct, are given in the Appendix.)

\section{Accuracy}

The solid lines in the six graphs in Figure 4 show the percentages of correct responses to targets in each of the 10 display positions. The first five graphs $(4 a-4 e)$ give the accuracy results for each subject; the sixth graph (4f) gives means, averaged across the five subjects. Mean accuracy varied from $98 \%$ correct responses, for targets in positions 


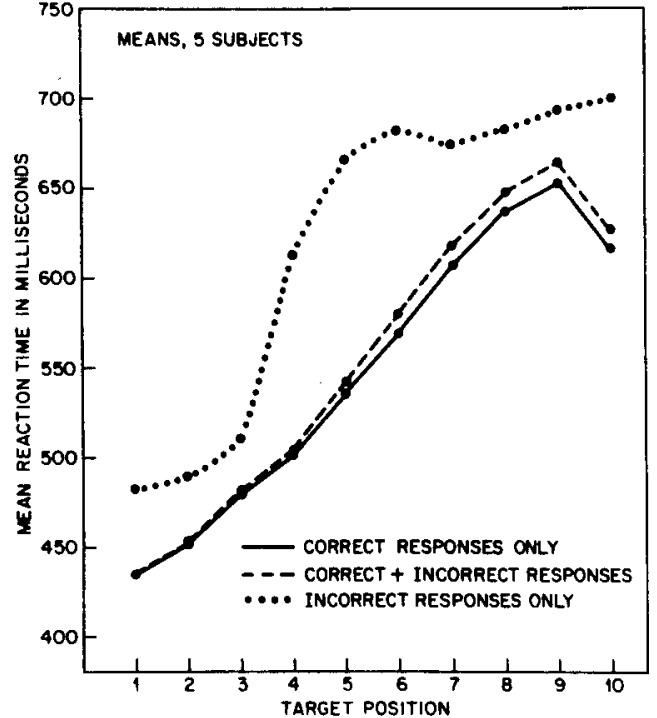

Figure 3. Mean RT data, averaged across the five subjects, for responses to each of the 10 display positions. The solid line shows mean RT for correct responses only, the dotted line shows mean RT for incorrect responses, and the broken line shows mean RT for all responses (correct plus incorrect).
2,3 , and 4 , to $67 \%$ correct, for targets in position 9 . Overall accuracy for individual subjects ranged from $94 \%$ for Subject J.L. to $80 \%$ for R.H. Every subject was more accurate in responding to position 2 than to position 1 ; four of the five subjects were more accurate to position 3 than position 1. Every subject was more accurate in responding to position 10 than to positions 8 and 9 .

The dotted lines in the first five graphs of Figure 4 show the best-fitting accuracy curves generated by the overlapping model, as determined by a least squares criterion. Only a single parameter, $\mathrm{A}$, was varied to make these fits; $\mathrm{k}, \lambda_{\mathrm{m}}$, and $\lambda_{\mathrm{n}}$ were left at the values determined by the fit to each subject's RT data. (The RT constant is not used in generating accuracy predictions.) The values found for $\mathrm{A}$ are given in Table 1. This table also gives the percentages of variance accounted for by the accuracy model, which ranged from $69 \%$ to $96 \%$ for the individual subjects. There is a rank-order correlation of $1.0(p<.02)$ between these percentages and the corresponding percentages for the fits to the RT data.

The dotted line in the last graph of Figure 4 shows the mean predicted accuracy curve. The mean predicted curve accounts for $94.6 \%$ of the variance in the mean accuracy data; standard error of prediction is 2.6 percentage points.

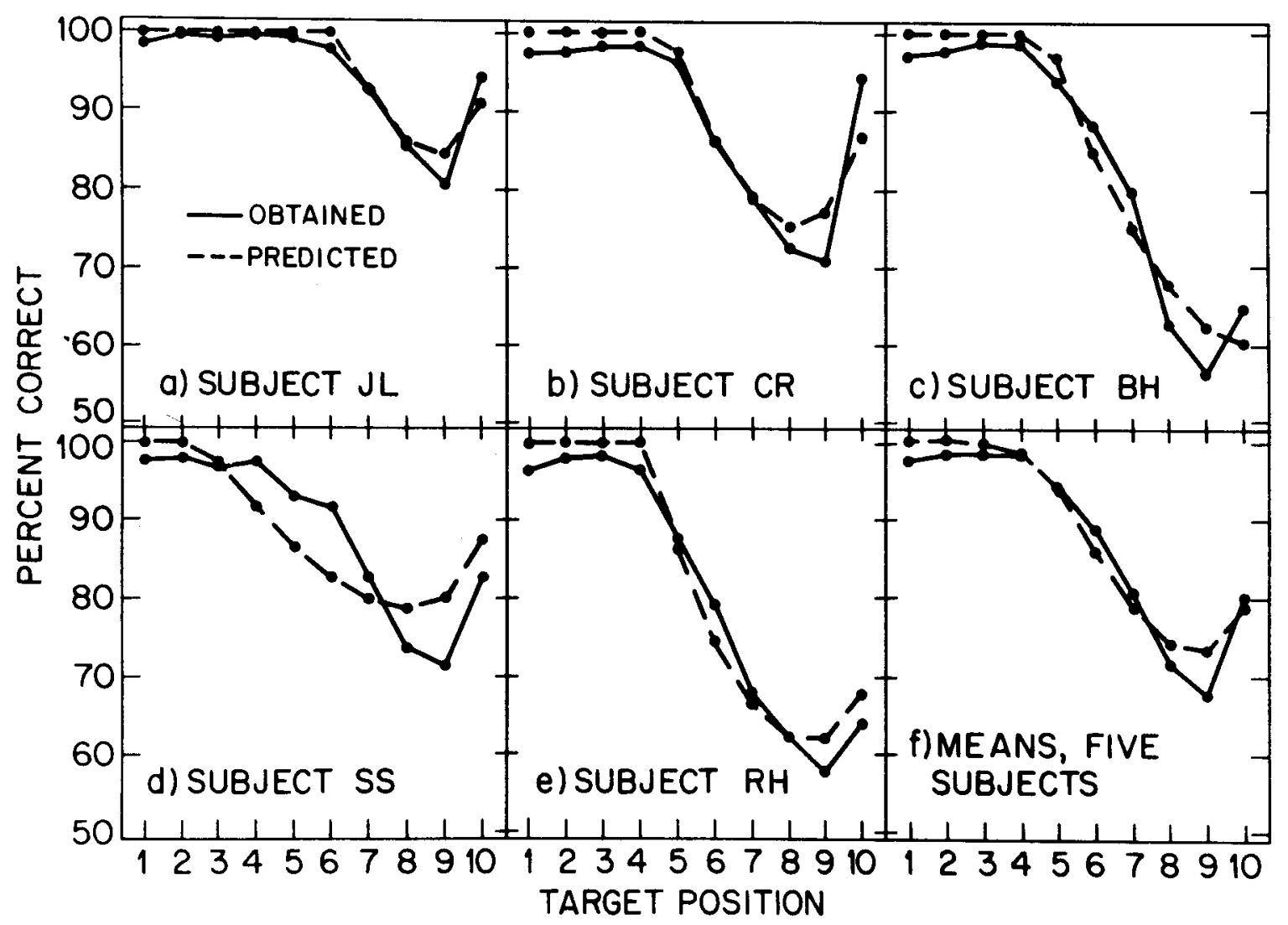

Figure 4. The solid lines show mean accuracy (percent correct) of responses to targets in each of the 10 display positions, for each of the individual subjects (4a-4e) and for the group as a whole (4f). The broken lines in $4 a-4 e$ show accuracy predictions generated by the overlapping model, using the model parameters obtained from the least squares fit to each subject's RT data. The values of $A$ used to generate these predictions, obtained by a least squares fit to each subject's accuracy data, are given in Table 1; the percentage of variance accounted for by these curves is also given in that table. The broken line in the last graph (4f) shows the mean predicted accuracy curve; this curve accounts for $95 \%$ of the variance in the group accuracy data. 


\section{No-Response Trials}

On rare occasion, a subject made no response at all within the 1.5-sec period allotted for the response. The proportion of trials on which this occurred was only .0028 of the total number of trials. These trials were not included in our analyses of RTs or of correct and incorrect responses.

\section{DISCUSSION}

Predictions generated by the overlapping model accounted for $98 \%$ of the variance in the group RT data for the 10 target positions and $95 \%$ of the variance in the group accuracy data. The model also did an excellent job of accounting for the individual RT data from all five of the subjects (variance accounted for averaged $95 \%$ ) and for the accuracy data from four of the subjects (variance accounted for averaged $93 \%$ for these four subjects, $88 \%$ for all five). By the usual standards, we can say that the overlapping model was quite successful in predicting serial-position effects for 10-letter arrays. Before we draw any conclusions from these results, however, some possible drawbacks must be considered.

\section{Is the Experiment an Adequate Test of the Model?}

If we had constructed the overlapping model to account for the data from the present experiment, the correspondence between the obtained serial-position curves and those generated by the model would be of little interest. The RT data are simple in form: an approximation to a straight line, monotonically increasing, with a downturn at the end. It would not be difficult to construct alternative models to "predict" an RT curve of this description. But the overlapping model was not constructed to fit these data; it was developed to account for the data from the gap experiment. The gap data bore no resemblance to a staight line with a downturn at the end-they didn't even have an end, since positions 8,9 , and 10 were not tested in that experiment. Thus, the predicted RT curves generated by the overlapping model are truly a priori: at the time the model was formulated, no data existed to test these predictions. It is in light of this fact that the good correspondence between the predicted and obtained curves should be considered.

The case for the model is further strengthened by the similarity between the predicted and obtained accuracy curves. The accuracy curves are more complex in shape than the RT curves; it would not be as easy to think of alternative models that would generate curves to fit these data. And, of course, the accuracy predictions are also a priori.

\section{The Number of Parameters}

The RT version of the overlapping model has four parameters, including the RT constant. However, only $\lambda_{m}$ and $\lambda_{n}$ affect the shape of the curve generated by the model-the monotonic, nearly linear increase, and (for most subjects) the turnover at the end. The parameter $k$ affects only the slope of the function: increases in $\mathrm{k}$ produce a steeper slope. (The slope is also affected by $\lambda_{m}$ : increases in $\lambda_{m}$ cause the slope to decrease.)

As for the RT constant, changes in this parameter simply raise or lower the entire function by that amount. We assume that there are many processes going on between stimulus and response that do not fall into the domain of the overlapping model. The length of time taken up by these processes is likely to vary from one subject to another; it will also depend upon factors such as the type of device used for the subjects' responses. Thus, overall RT will vary between subjects and between experiments, and it is necessary to adjust the RT constant in order to compensate for these variations.

It is of interest to note that the RT constants derived from the fits to the subjects' data in this experiment were the only model parameters that differed noticeably from the parameters found in the gap experiment (Harris et al., 1979). The values found in the gap experiment for $\mathrm{k}, \lambda_{\mathrm{m}}$, and $\lambda_{\mathrm{n}}$ were $1.14, .84$, and .63 , respectively; these values all fall well within the range of values found for these parameters in the present experiment (see Table 1). The value of $A$ reported by Harris et al. was 104; this again falls within the range of the values found here. Even the RT constant did not differ by much: $385 \mathrm{msec}$ for the gap experiment versus an average of $360 \mathrm{msec}$ in the present experiment. This relative lack of variation is somewhat surprising, since the two experiments involved different subjects and different equipment (the gap experiment was carried out on a tachistoscope, whereas the present experiment used computer-driven video monitors).

The accuracy parameter. The ability of the overlapping model to predict accuracy results is particularly noteworthy in view of the fact that these predictions depend on only a single parameter, $A$. (The three other parameters used in generating accuracy predictions remained at the values determined by the fits to the RT data.) According to the new conceptualization of the accuracy version of the model, discussed in the introduction, the parameter A gives an indication of the processing accuracy of a given subject: how likely it is that the processing performed in a given amount of time will be inaccurate, and how quickly this likelihood increases as processing continues. Smaller values of A suggest lower accuracy per unit of time and/or a more rapid decline in accuracy.

\section{Systematic Differences between Predicted and Obtained Curves}

Although the overlapping model's predictions account for an impressive proportion of the variance in the serialposition data, there are some systematic differences in shape between the theoretical curves and the obtained ones. These differences can be seen in both the RT and the accuracy curves.

Early serial positions. The first of these differences, 
which can be seen most clearly in the group RT data (Figure 2f), involves the shape of the curve for the first seven or eight target positions. That part of the model's theoretical curve is nearly linear, whereas the obtained curve is slightly higher at the left end, slightly lower in the middle. These discrepancies between the obtained and predicted functions are also visible in the individual RT data from four of the five subjects (Figures $2 \mathrm{a}-\mathrm{d}$ ). Notice that the graph of the group RT data would be nearly linear for the first seven target positions if the subjects' responses for the first three or four positions, and especially for the first position, had been quicker. ${ }^{8}$ Notice, too, that obtained performance for the first three or four positions is poorer than the model's predictions in both the RT data and the accuracy data. The highest accuracies were obtained, not for targets in position 1 , but for positions 2 and 3.

Two possible explanations can account for these results. The simplest one is that they are due to a failure to maintain fixation. Our subjects were highly motivated to respond as quickly and as accurately as possible. For this reason, it was necessary to remind them frequently about the importance of maintaining fixation, since they could actually improve their overall performance by fixating a little to the right of the fixation point, on position 2 or 3 , or even on 4 or 5 . A tendency to do this occasionally would result in a slight worsening of performance on the first two or three display positions, relative to the later positions. This is just what was observed.

A second, more interesting possibility is that scan speed may not have been constant across the display, but may have been more rapid at the left end than at the right. (This, again, would produce the observed results.) Harris et al. postulated a scanning mechanism that could not vary its speed within a trial; this postulate was grounded on earlier evidence that processing strategies could not be adjusted within a trial on the basis of information provided by the display itself. But a different assumption, still consistent with the earlier evidence, is possible: perhaps the rate of scan can be programmed in advance to be more rapid over the early display positions and to slow down somewhat for the more difficult later ones. Such a processing strategy might be a highly effective one, and could have been learned and practiced by our subjects during their long period of training.

Late serial positions. Systematic deviations between theoretical and obtained curves are also noticeable at the right end of the functions. The graphs of the group data seen in Figures $2 \mathrm{f}$ and $4 \mathrm{f}$ hit a high point (or, in the case of accuracy, a low point) at position 9 and then show a sharp reversal of direction. The predictions generated by the model show, instead, a gentle curve. The overlapping model in its present form cannot generate curves with sharp peaks and sudden changes of direction.

It would, of course, be possible to produce theoretical curves that are closer in shape to the obtained ones by adding additional features-and additional parameters-to the model. We do not choose to adopt that alternative.
The overlapping model in it present version can account for many of the phenomena of visual search. It can generate predicted serial-position curves that provide a good, though not perfect, match for serial-position curves obtained long after those predictions were made. These results give strong support for the view that the processing of individual characters in visual search overlaps in time.

\section{Conclusion}

There are data suggesting that the individual items in a display are processed in a particular order. Such data present difficulties for parallel models of visual search. There are also data indicating some kind of mutual interference between adjacent display items, which suggests that these items are being processed simultaneously. Such data present difficulties for serial models of visual search. Overlapping models, such as the model discussed here (we know of no other examples at present), can account for both types of results. Overlapping processing involves both an order of processing and simultaneous processing of nearby characters.

Overlapping is a manner of processing that falls between serial and parallel. Serial and parallel processing can be seen as special cases of overlapping: zero overlap and complete overlap. In the past, researchers in information processing have tried to ascertain by experimental means whether processing was serial or parallel. The results of some of these experiments (e.g., Sperling, 1970; Weisstein, 1966) have seemed inconclusive because they fell between the serial prediction and the parallel prediction. Such results can be viewed as evidence for overlapping processing.

The overlapping model discussed here was designed to apply to a particular situation: when the task is to search for a target character or characters in a briefly presented display consisting of several characters. The nature of this task demands that the unit of processing be the individual character. Thus, according to the model, characters enter the processor one at a time. In a different task-ordinary reading, for example-the unit of processing might be a larger "chunk," such as a word or a syllable. Evidence suggesting that reading may involve overlapping processing of words or parts of words has existed for a long time-since Buswell's (1920) discovery of what he called the eye-voice span. Buswell found that people reading aloud keep their eyes moving along the text several words ahead of the word they are saying. We know that the eyes' point of fixation shifts every $250 \mathrm{msec}$ or so. With each fixation, a new chunk of text enters processing. Meanwhile, processing of the preceding chunks continues. Eventually, processing of a given chunk will be completed and the result will be a spoken word; but in the interim, several more fixations will have occurred and several more chunks will have entered the processor. Processing of this sort-overlapping processing-is smooth, continuous, and highly efficient. 


\section{REFERENCES}

Buswell, G. T. (1920). An experimental study of the eye-voice span in reading. Supplemental Educational Monographs, Whole No. 17.

EsTES, W. K., \& WOLFORD, G. L. (1971). Effects of spaces on report from tachistoscopically presented letter strings. Psychonomic Science, 25, 77-80.

HaRRIS, J. R., Shaw, M. L., \& Bates, M. (1979). Visual search in multicharacter arrays with and without gaps. Perception \& Psychophysics, 26, 69-84.

SHAw, M. L. (1977). Reaction time in reading a tachistoscopic display for a memory set item. Perception \& Psychophysics, 21, 15-25.

SHAw, M. L. (1978). A capacity allocation model for reaction time. Journal of Experimental Psychology: Human Perception and Performance, 4, 586-598.

SPERLING, G. (1970). Short-term memory, long-term memory, and scanning in the processing of visual information. In F. A. Young \& D. B. Lindsley (Eds.), Early experience and visual information processing in perceptual and reading disorders. Washington, DC: National Academy of Sciences.

StERnBERG, S. (1967, April). Scanning a persisting visual image versus a memorized list. Paper presented at the meeting of the Eastern Psychological Association, Boston.

STERnBERG, S. (1969). The discovery of processing stages: Extensions of Donders' method. In W. G. Koster (Ed.), Attention and performance II. Amsterdam: North Holland. (Reprinted from Acta Psychologica, 1969, 30, 276-315)

Weisstein, N. (1966). Backward masking and models of perceptual processing. Journal of Experimental Psychology, 72, 232-240.

ZaJoNC, R. B. (1976). Family configuration and intelligence. Science, $192,227-236$.

\section{NOTES}

1. A good way to gain a quick understanding of the overlapping model is to think of it in terms of an analogy. Imagine that the task is not to process an array of alphanumeric characters, but rather to rear several children from birth to adulthood. Theoretically, this can be accomplished serially-one child is born and reared to adulthood, and then the next child is born. Or it can be accomplished in parallel-twins, triplets, and so on. The third alternative is the way childrearing in multichild families is usually carried out: by overlapping processing. Overlapping processing produces serial-position effects that, in this case, are called birth-order effects; these effects often bear a striking resemblance to those obtained in information-processing experiments (see, e.g., Zajonc, 1976). The analogy holds up even in regard to the asymmetry of the effects of overlapping on processing efficiency: parents do not have unlimited processing capacity, so the processing of a given child is adversely affected by the presence of other children in the home. But since babies require more parental processing capacity than older children, a given child is affected more by competition from later-born siblings than by competition from the siblings who were born previously. Note that total "processing efficiency," in the overlapping model, does not equal a constant when summed over items. Just as in the multichild home, the total amount of work performed is greater when there are many items than when there are few.
2. We assume that only the encoding stage is affected by factors such as serial position and retinal locus. For the other stages of information processing, which Sternberg (1969) has labeled "serial comparison," "binary decision," and "response organization," we assume a constant processing time per item that is unaffected by visibility factors, array size, or serial position within the array. The amount of time required for these three stages is represented in the model by a fourth parameter, which we call the RT constant. The RT constant is the baseline, or intercept, of the predicted RT function.

3. The proportion $A / T_{i}$ can go above 1.0, whereas a subject's performance cannot. We interpreted cases in which $A / T_{i}>1.0$ as predictions of errorless performance. The corresponding interpretation for cases in which $A / T_{i}<.50$ (chance in these experiments) would also be reasonable, but was unnecessary because estimates of $A / T_{i}$ invariably exceeded .50 .

4. The RT serial-position curve that would be predicted by this accuracy model is not perfectly flat; it goes up at the rate of $10 \mathrm{msec}$ per item due to the postulated rate of scan.

5. For one subject, C.R., the total number of experimental trials was 1,248 per target position; for the other four subjects, there were 1,200 trials per target position. A portion of the data from one subject, J.L., was lost due to computer malfunction; the results reported for this subject are based on a minimum of 960 trials per target position.

6 . The formula for the standard error of prediction is:

$$
\left[\sum_{i=0}^{N}\left(y_{o_{i}}-y_{p_{i}}\right)^{2} / N\right]^{1 / 2},
$$

where $y_{0_{i}}$ is the obtained value, $y_{p_{i}}$ is the predicted value, and $N$ is the number of points involved (in this case, 10 ).

7. Model fits to RT data are generally done with data from correct responses only; this was the method used by Harris et al. (1979). However, it might be argued that since the overlapping model deals with accuracy as well as with RT, the RT predictions should be fit to all responses, rather than correct ones alone. In the case of the present experiment, such a procedure would produce results very similar in appearance to those shown in Figure 2. The percentage of variance in the group data accounted for by the mean predicted RT curve would be 97.0 (as opposed to 97.9 when only correct responses are considered).

8. One possible way of dealing with the slight elevation at the left end of the obtained RT curves is by employing a "correction factor"- - a fifth parameter that produces a similar elevation at the left end of the predicted functions. When we tried this, by adding to the predicted RT values the quantity $C / i$ (where $C \geq 0$ and is determined by a least squares fit to each subject's data, and $i$ is the index of serial position), we naturally achieved somewhat better fits to the individual subjects' RT data. Since using an additional parameter will almost inevitably produce a better fit, this is not very interesting; nor does the relatively small improvement in the fits to the RT data justify the use of this additional parameter. But what is interesting, and quite unexpected, is that "correcting" the elevation of the left end of the RT functions led to an improvement in the accuracy fits as well: the values of $k, \lambda_{m}$, and $\lambda_{n}$ derived from this procedure produced better accuracy fits for the three subjects whose accuracy fits had been the poorest, particularly Subject S.S. (whose fit went from $69.2 \%$ to $80.9 \%$ in variance accounted for). For the five subjects, respectively, variance accounted for by the accuracy fits was $91.9 \%, 89.8 \%, 94.1 \%, 80.9 \%$, and $96.3 \%$. 


\section{APPENDIX}

Mean Reaction Time (in Milliseconds) and Accuracy Data for Five Subjects and 10 Target Positions

\begin{tabular}{|c|c|c|c|c|c|c|c|c|c|c|c|}
\hline \multirow{2}{*}{ Condition } & \multicolumn{10}{|c|}{ Target Position } & \multirow{2}{*}{$\begin{array}{l}\text { Mean of } \\
10 \text { Positions }\end{array}$} \\
\hline & 1 & 2 & 3 & 4 & 5 & 6 & 7 & 8 & 9 & 10 & \\
\hline \multicolumn{12}{|c|}{ Subject J.L. } \\
\hline RT, Correct Responses Only & 446 & 460 & 496 & 508 & 543 & 560 & 610 & 647 & 678 & 614 & 556 \\
\hline RT, Incorrect Responses Only & 413 & 466 & 458 & 654 & 755 & 767 & 729 & 752 & 777 & 754 & 652 \\
\hline RT, All Responses & 446 & 460 & 495 & 509 & 545 & 565 & 619 & 663 & 698 & 622 & 562 \\
\hline Percent Correct & 98.5 & 99.6 & 99.2 & 99.5 & 99.2 & 97.9 & 92.9 & 85.5 & 80.4 & 94.2 & 94.7 \\
\hline \multicolumn{12}{|c|}{ Subject C.R. } \\
\hline RT, Correct Responses Only & 452 & 472 & 499 & 507 & 550 & 599 & 643 & 667 & 682 & 600 & 567 \\
\hline RT, Incorrect Responses Only & 474 & 479 & 527 & 537 & 675 & 722 & 723 & 722 & 726 & 744 & 633 \\
\hline RT, All Responses & 453 & 472 & 500 & 507 & 555 & 617 & 660 & 682 & 695 & 609 & 575 \\
\hline Percent Correct & 97.4 & 97.5 & 98.1 & 98.2 & 96.1 & 85.9 & 79.0 & 72.4 & 70.7 & 94.0 & 88.9 \\
\hline \multicolumn{12}{|c|}{ Subject B.H. } \\
\hline RT, Correct Responses Only & 411 & 416 & 432 & 456 & 476 & 500 & 532 & 574 & 583 & 582 & 496 \\
\hline RT, Incorrect Responses Only & 468 & 489 & 445 & 556 & 553 & 586 & 598 & 590 & 597 & 593 & 547 \\
\hline RT, All Responses & 413 & 418 & 432 & 457 & 480 & 510 & 546 & 580 & 589 & 586 & 501 \\
\hline Percent Correct & 97.0 & 97.6 & 98.8 & 98.5 & 93.9 & 88.2 & 79.7 & 62.6 & 56.3 & 64.6 & 83.7 \\
\hline \multicolumn{12}{|c|}{ Subject S.S. } \\
\hline RT, Correct Responses Only & 447 & 443 & 454 & 460 & 473 & 493 & 508 & 530 & 548 & 517 & 487 \\
\hline RT, Incorrect Responses Only & 512 & 536 & 537 & 515 & 587 & 556 & 560 & 567 & 569 & 591 & 553 \\
\hline RT, All Responses & 448 & 445 & 457 & 462 & 481 & 498 & 517 & 539 & 554 & 530 & 493 \\
\hline Percent Correct & 97.8 & 98.0 & 96.7 & 97.5 & 93.2 & 91.7 & 82.8 & 73.7 & 71.4 & 82.7 & 88.5 \\
\hline \multicolumn{12}{|c|}{ Subject R.H. } \\
\hline RT, Correct Responses Only & 417 & 469 & 524 & 578 & 636 & 692 & 740 & 767 & 766 & 768 & 636 \\
\hline RT, Incorrect Responses Only & 545 & 474 & 582 & 801 & 759 & 785 & 769 & 786 & 803 & 826 & 713 \\
\hline RT, All Responses & 422 & 469 & 525 & 586 & 651 & 711 & 749 & 774 & 782 & 789 & 646 \\
\hline Percent Correct & 96.5 & 97.9 & 98.3 & 96.5 & 87.6 & 79.1 & 67.8 & 62.2 & 57.7 & 63.8 & 80.7 \\
\hline \multicolumn{12}{|c|}{ Mean of Five Subjects } \\
\hline RT, Correct Responses Only & 435 & 452 & 481 & 502 & 536 & 569 & 607 & 637 & 652 & 616 & 549 \\
\hline RT, Incorrect Responses Only & 482 & 489 & 510 & 613 & 666 & 683 & 676 & 683 & 694 & 701 & 620 \\
\hline RT, All Responses & 436 & 453 & 482 & 504 & 542 & 580 & 618 & 648 & 663 & 627 & 555 \\
\hline Percent Correct & 97.4 & 98.1 & 98.2 & 98.0 & 94.0 & 88.6 & 80.4 & 71.3 & 67.3 & 79.8 & 87.3 \\
\hline
\end{tabular}

(Manuscript received March 6, 1985;

revision accepted for publication July 10, 1985.) 